CARDIOVASCULAR MEDICINE

\title{
Highly accurate coronary angiography with submillimetre, 16 slice computed tomography
}

\author{
G J Morgan-Hughes, C A Roobottom, P E Owens, A J Marshall
}

Heart 2005;91:308-313. doi: 10.1136/hrt.2004.034892

See end of article for authors' affiliations ....................

Correspondence to: Dr Gareth J MorganHughes, Cardiology Department, SWCC, Plymouth NHS Trust, Derriford, Plymouth' PL6 8DH, UK; gareth. morgan-hughes@phnt. swest.nhs.uk

Accepted 15 May 2004
Objective: To assess submillimetre coronary computed tomographic angiography (CTA) in comparison with invasive quantitative coronary angiography as the gold standard and to examine the effect of significant coronary artery calcification (CAC), which is known to impede lumen visualisation, on the accuracy of the examination.

Methods: After invasive coronary angiography, 58 patients underwent coronary imaging with a GE Lightspeed 16 computed tomography (CT) system. CAC was quantified after an ECG triggered acquisition with a low tube current. Coronary CTA was performed with retrospective ECG gating and a $16 \times 0.63 \mathrm{~mm}$ collimation and was reconstructed with an effective 65-250 ms temporal resolution. All 13 major coronary artery segments were evaluated for the presence of $\geqslant 50 \%$ stenosis, and compared with the gold standard.

Results: One patient moved and could not be evaluated. All segments (except occluded segments) were evaluated for 57 patients. Overall the accuracy of coronary CTA for detection of $\geqslant 50 \%$ stenosis was: sensitivity $83 \%$, specificity $97 \%$, positive predictive value $80 \%$, and negative predictive value $97 \%$. The number of diseased coronary arteries was correctly diagnosed in 34 of 38 (89\%) patients overall. Altogether 21 of 57 (37\%) patients had a CAC score $\geqslant 400$, which was predefined as representing significant CAC. Excluding these patients from the analysis improved the accuracy of coronary CTA to a sensitivity of $89 \%$, specificity $98 \%$, positive predictive value $79 \%$, and negative predictive value $99 \%$.

Conclusions: Non-invasive coronary angiography with submillimetre CT is reliable and accurate. It appears that a subgroup of patients may be selected based on CAC score in whom the investigation has even higher accuracy. Coronary CTA has reached the stage where it should be considered for a clinical role. Further research is required to define this role.
$\mathrm{T}$ he emergence of ECG gated multislice computed tomography (CT) has stimulated great interest among cardiologists. This is primarily because this new technology may provide a clinically useful method for performing non-invasive coronary angiography. Four slice CT has been extensively evaluated in this context and is lacking in both reliability of image quality and accuracy of results. ${ }^{1-5}$ However, these studies have identified certain patient related factors that appear to affect the provision of a clear image of the contrast enhanced coronary artery lumen. Specifically, high heart rates induce motion artefact and excessive coronary artery calcification (CAC) impedes accurate lumen visualisation and may produce blooming artefacts. ${ }^{6}$

Recently four slice CT has been superseded by 16 slice CT. For cardiac applications this technology provides not only a submillimetre collimation but also improved temporal resolution. Preliminary studies have been performed with this type of technology. ${ }^{7-9}$ Although the results of these studies have been favourably received, improvements are still needed before clinical use. ${ }^{10}$ Potential room for this improvement exists both in the image acquisition and in reconstruction protocols but also perhaps in patient selection. Both of the previously reported studies used a $12 \times 0.75 \mathrm{~mm}$ slice collimation and provided a $210 \mathrm{~ms}$ temporal resolution. ${ }^{78}$ Coronary computed tomographic angiography (CTA) may now be performed with higher specification than this. An acquisition collimation of $16 \times 0.625 \mathrm{~mm}$ provides spatial resolution in the long axis, which approaches that of the inplane resolution. Using acquired data from two or more cardiac cycles to reconstruct an image (partial subsegmental reconstruction) may reduce the effective temporal resolution to an estimated $125 \mathrm{~ms}$ or less. In addition a larger number of post-processing techniques are now available for image analysis, although it should be stressed that the benefit of these is unproved (fig l).

The primary intention of this study was to assess the accuracy of 16 slice, submillimetre coronary CTA, in comparison with the ideal standard, invasive coronary angiography. The secondary aim was to ascertain the precise effect of a significant level of CAC (defined as a CAC score $\geqslant 400$; see below) on the accuracy of the technique described.

\section{METHODS \\ Patients}

This was a prospective observational study carried out between May and October 2003. Patients undergoing day case coronary angiography were approached with a view to undergoing CAC scoring and coronary CTA at a later date for comparative purposes. The multislice CTs were recorded within one month of the invasive angiograms. Consecutive patients, fulfilling the appropriate criteria, were approached until all of the scanning slots were filled. A total of 58 patients (mean (SD) age 61 (11) years, mean (SD) heart rate 61 (8) beats/min, mean (SD) body mass index 30 (3), 47 men, 11 women) were studied. Patients were included only if they were capable of a minimum 20 second breath hold and had a regular, resting heart rate of $\leqslant 65$ beats/min. Heart rate was recorded at the time of the invasive coronary

\footnotetext{
Abbreviations: CAC, coronary artery calcification; $\mathrm{CT}$, computed tomography; CTA, computed tomographic angiography
} 

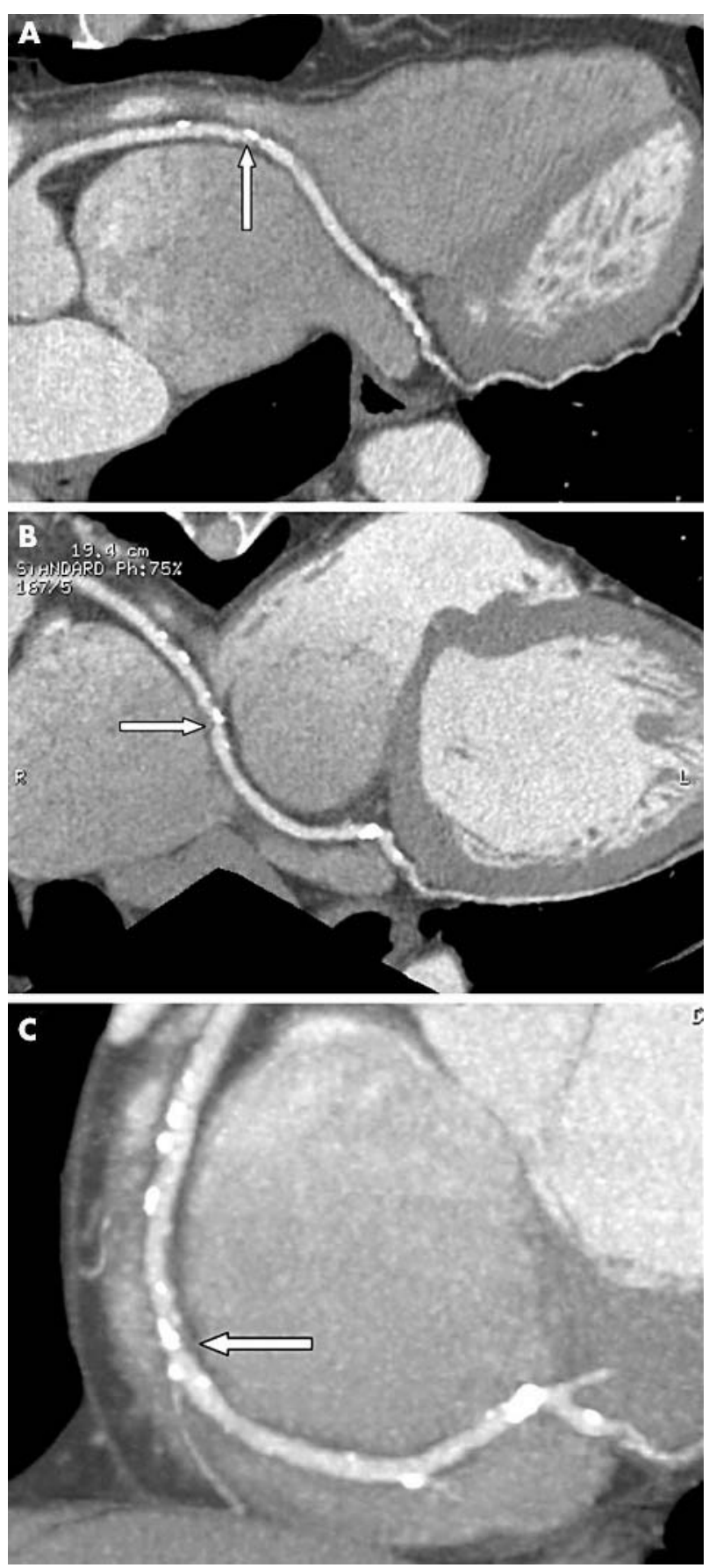

Figure 1 Submillimetre coronary computed tomographic angiography (CTA) is shown with a type of curved multiplanar reconstruction known as vessel analysis (reconstructed slice thickness $1 \mathrm{~mm}$ ). Post-processing has generated these two dimensional angiograms, which are in the plane of the right coronary artery, showing the vessel to the point of bifurcation and distally, the posterior left ventricular branch. Panels $A$ and $B$ show the right coronary artery in a cross sectional orientation, and panel $C$ in a longitudinal orientation. This type of post-processing allows for accurate stenosis assessment. Minor areas of atheroma and calcification are shown, one of which is associated with $>50 \%$ diameter reduction of the lumen (arrows).

angiogram. Exclusion criteria were previous coronary artery bypass graft surgery, allergic reaction to contrast agent, age $<50$ years, severe renal failure, or an unstable clinical condition. The local research ethics committee approved the study, and all patients gave written informed consent.
Cardiac catheterisation and coronary angiography

Cardiac catheterisation and coronary angiography was carried out with digital fluoroscopy systems (Integris Allura; Philips Medical Systems, Reigate, Surrey, UK) according to traditional techniques by consultant cardiologists or by cardiology trainees supervised by consultant cardiologists. Multiple views of the coronary arteries were obtained. The coronary angiogram was reported in standard fashion by the operating cardiologist. In the case of trainee examinations this was in conjunction with a consultant cardiologist. The comparative investigation examined all major coronary artery segments and side branches segments (segments 1 to 13 of the American Heart Association model). ${ }^{11}$ Any of these segments of the coronary artery for comparative evaluation reported to contain a stenotic lesion (or occlusion) of $>30 \%$ were evaluated by quantitative coronary angiography in two orthogonal views. Angiographic stenosis was graded as maximum lumen diameter loss for the two views. Totally occluded vessels were not assessed beyond the occluded segment. One entirely recessive right coronary artery was not assessed. Segments of coronary artery containing stents were evaluated separately. After coronary angiography with or without quantitative coronary angiography each coronary artery segment was declared either "normal or $<50 \%$ stenotic" or " $\geqslant 50 \%$ stenotic". No attempt was made to make a comparative evaluation of less severe lesions.

\section{Multislice CT coronary artery imaging acquisition}

Multislice CTs were acquired with a GE Lightspeed 16, submillimetre, 16 slice CT system (General Electrical Medical Systems, Milwaukee, Wisconsin, USA). To enable both prospective and retrospective ECG gating the ECG was recorded continuously throughout the preparation and image acquisition period. An 18 gauge antecubital vein catheter was inserted for contrast administration.

The same general acquisition process, to which there were four stages, was followed each time. Each stage of the acquisition was preceded by the same gentle hyperventilation protocol, which took 19 seconds. Stage 1 was an anteroposterior and lateral scout acquisition performed to ensure that the heart was well centred and to allow the acquisition volume to be estimated. In stage 2 , the initial non-contrast CT of the heart, for coronary calcification scoring, the volume extended from below the carina to the apex of the heart. Acquisition parameters were prospective ECG triggering at $60 \%$ of the RR interval, $500 \mathrm{~ms}$ gantry rotation, $4 \times 2.5 \mathrm{~mm}$ collimation, $80 \mathrm{~mA}$, and $120 \mathrm{kV}$. To minimise the increase in the total effective patient radiation dose, this stage of the scanning process was conducted with a low tube current (80 mA). A total CAC score was obtained only to enable division of patients into two groups, with either a high (Agatston score $\geqslant 400$ ) level of CAC or not. This cut off point was used because it acceptably defines a high risk score, although it should be noted that the acquisition protocol described here differs from that originally described by Agatston et al. ${ }^{13}$

Before the final stage the transit time was established with a test bolus of $20 \mathrm{ml}$ of contrast (Iomeron 400, Bracco, Milan, Italy or Iopromide, Ultravist 370, Schering, Berlin, Germany) flushed by $50 \mathrm{ml}$ of saline, both given at $4 \mathrm{ml} / \mathrm{s}$ (stage 3 ). Stage 4 was contrast enhanced coronary CTA. Acquisition and initial reconstruction parameters varied according to the patient's heart rate. Three basic protocols were used; table 1 describes the full acquisition parameters. For patients with a heart rate $<60$ beats/min, images were reconstructed from information taken from one cardiac cycle, providing a $250 \mathrm{~ms}$ temporal resolution (partial segment reconstruction). For patients with either a heart rate of between $60-75$ beats/min 
Table 1 Acquisition protocol for submillimetre coronary computed tomographic angiography (CTA)

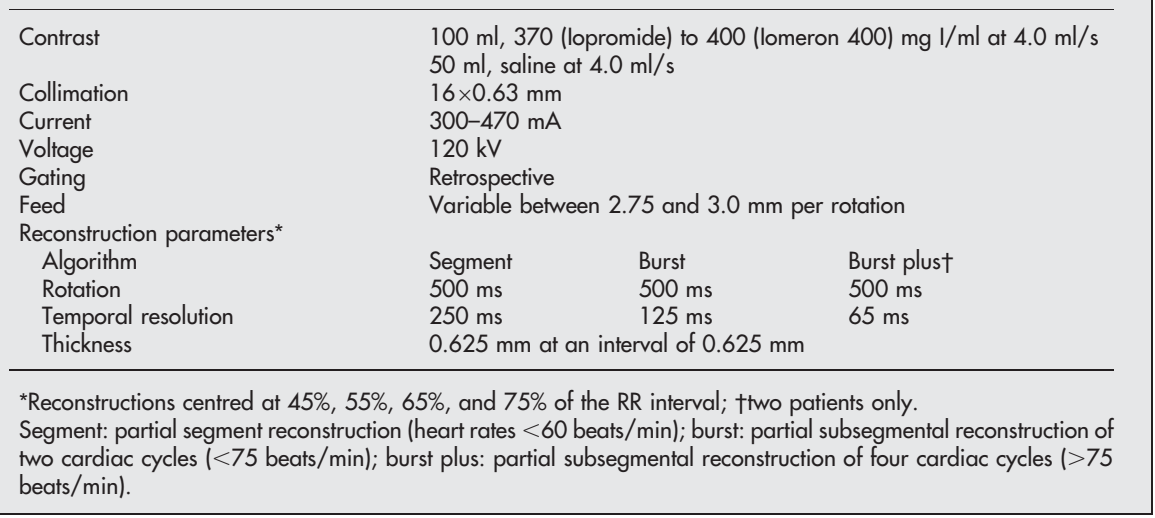

or a heart rate $>75$ beats/min, images were reconstructed from more than one cardiac cycle (partial subsegmental reconstruction). For heart rates between $60-75$ beats/min two cardiac cycles were used providing a temporal resolution of $125 \mathrm{~ms}$. This was the predominant mode of reconstruction. For heart rates $>75$ beats/min four cardiac cycles were used providing a temporal resolution estimated at $65 \mathrm{~ms}$. Constant acquisition parameters were the use of retrospective ECG gating, the detector collimation $(16 \times 0.63 \mathrm{~mm})$, and the tube voltage $(120 \mathrm{kV})$. The feed was $2.75-3.0 \mathrm{~mm}$ for each rotation depending on the patient's heart rate (faster heart rate, faster feed). The tube current (300-470 mA) was varied automatically according to body size. Contrast was enhanced by injecting $100 \mathrm{ml}$ of contrast agent (Iomeron 400 or Iopromide) at $4 \mathrm{ml} / \mathrm{s}$, flushed with $50 \mathrm{ml}$ of saline at $4 \mathrm{ml} / \mathrm{s}$. The mean (SD) delay between initiation of the contrast injection and scan initiation was 23 (6) seconds.

\section{Multislice CT coronary imaging reconstruction and evaluation \\ CAC scoring}

The images were transferred to the workstation for CAC quantification with Smart score software (CardIQ Analysis II software, Advantage Windows 4.1 Workstation, General Electrical Medical Systems). The CTs were evaluated by consensus of two investigators (GMH and CAR) blinded to the results of the invasive coronary angiograms and experienced in the technique of coronary CTA. For the purposes of this study the CAC was quantified by the method originally described by Agatston et $a l^{13}$ but adapted for low tube current multislice CT. Therefore, any tissue above the threshold of $130 \mathrm{HU}$ (occupying a minimum area of $0.5 \mathrm{~mm}^{2}$ to avoid confusion with image noise) was identified by the application software and highlighted. Any area highlighted, which clearly represented CAC, was identified as a region of interest. Sequential axial sections were analysed. The total calcified area (the score) was then calculated by the workstation software.

\section{Coronary CTA}

The mean (SD) breath hold time was 20 (2) seconds. Images were reconstructed (initial reconstruction thickness $0.625 \mathrm{~mm}$ at an interval of $0.625 \mathrm{~mm}$ ) from diastolic data sets centred at $45 \%, 55 \%, 65 \%$, and $75 \%$ of the RR interval. These images were transferred to a workstation for motion artefact assessment (CardIQ Analysis II software). Further images, offset to the beginning and end of the RR interval by $10 \%$ increments (and if need be $5 \%$ ), were reconstructed if motion artefact was problematic. A maximum of 10 images for each patient were reconstructed. If more than
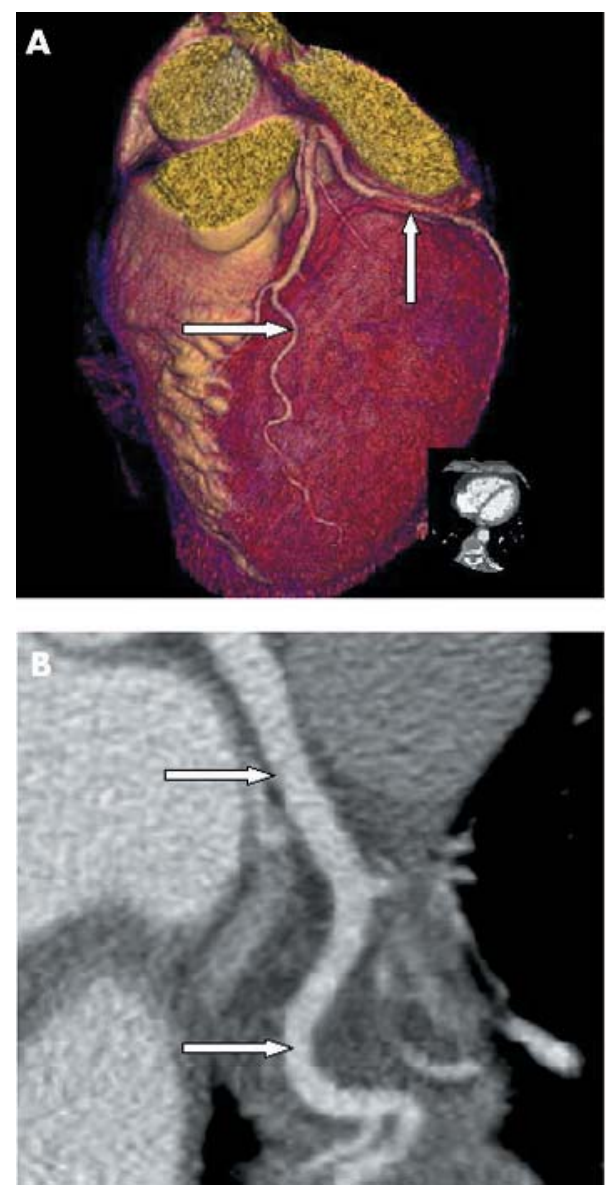

Figure 2 (A) Submillimetre coronary CTA with a three dimensional volume rendered reconstruction. The left anterior descending artery (horizontal arrow) is shown with two small diagonal branches and a larger septal branch is visible in this view. The circumflex (vertical arrow) is shown with the great cardiac vein, partially removed with volume rendering techniques, passing over the top. In this case all 13 segments of coronary artery were correctly reported as normal, although it should be noted that no judgement on the diagonal branches was based solely on this three dimensional volume rendered reconstruction.

(B) Submillimetre coronary CTA with a curved multiplanar reconstruction of a proximal circumflex (top arrow) and large first obtuse marginal (bottom arrow). All segments were normal.

four images were reconstructed then the four with the least artefact were selected for further post-processing and scan evaluation. 

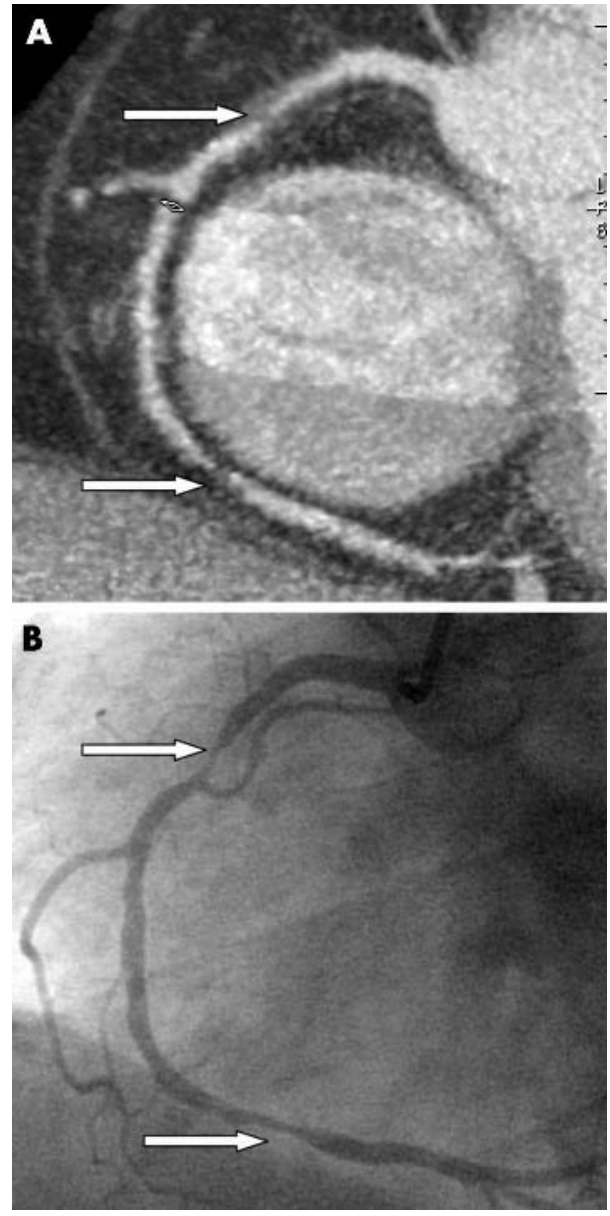

Figure 3 (A) Submillimetre coronary CTA of the right coronary artery, reconstructed by curved multiplanar reconstruction (vessel analysis), and (B) the comparative invasive coronary angiogram. The coronary CTA has a moderate amount of both image noise and motion artefact. Two lesions (arrows, panel B) were causing $>50 \%$ stenosis on quantitative coronary angiography of the invasive angiogram, both of which were identified correctly by coronary CTA (arrows, panel A).

Coronary CTAs were assessed simultaneously with the four previously reconstructed diastolic phases with the least motion artefact. The CardIQ Analysis II software allows not only simultaneous viewing of four diastolic phases but also simultaneous (or rapidly interchangeable) use of multiple post-processing techniques. Automatic (and manual) volume rendering techniques (fig 2) were generally used to ascertain the morphology and course of the vessels. Overall evaluation relied heavily on interactive viewing of multiplanar reconstructions (fig 2 ) and maximum intensity projections. If need be, further evaluation and quantification of coronary artery stenoses depended on a specific post-processing software package, based on curved multiplanar reconstruction, known as vessel analysis (fig 1 ).

All segments, including those with suboptimal image quality, were evaluated and judged for the presence or absence of $a \geqslant 50 \%$ lesion according to the invasive coronary angiograms (fig 3). Any segments clearly containing coronary artery stents were assessed separately for stent patency and significant in-stent restenosis. If total vessel occlusion was suspected then this was recorded (fig 4). Although comparative analysis of any segments distal to a total occlusion was not planned, these segments were evaluated in case the multislice CT finding of an occluded segment had been incorrect.
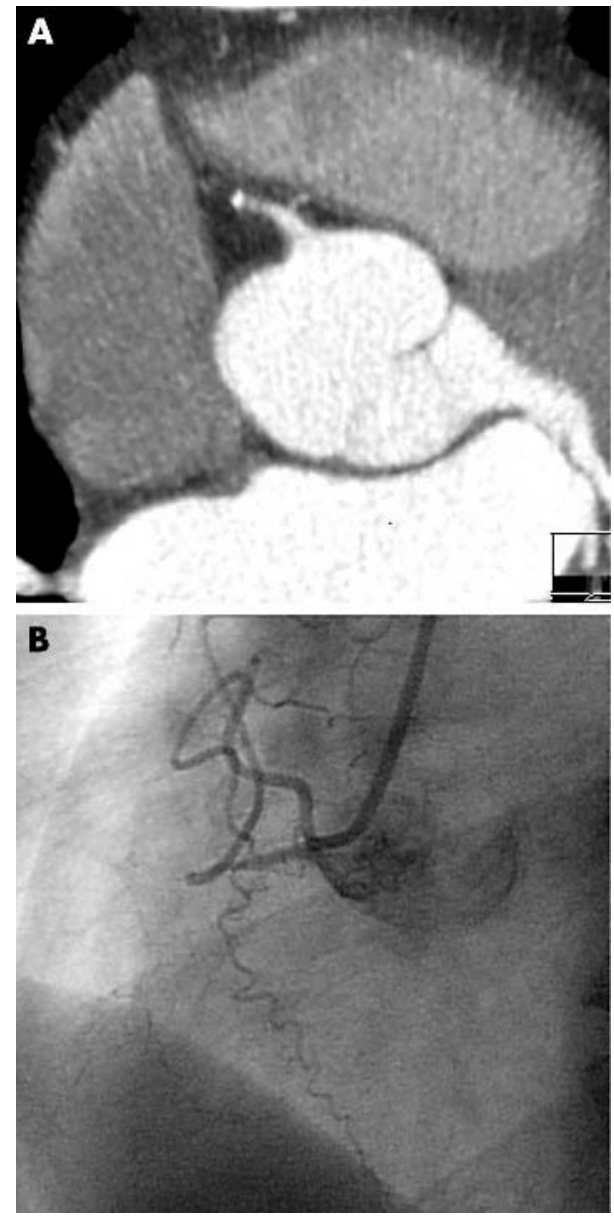

Figure 4 (A) Submillimetre coronary CTA of the right coronary artery, reconstructed as a simple axial section, and (B) the comparative invasive coronary angiogram. Both imaging modalities show proximal right coronary artery occlusion.

Standard descriptive statistics were used. Results of the two angiographic techniques were then compared, with the invasive coronary angiogram acting as the yardstick. This generated true or false, positive or negative findings for each coronary artery segment assessable with both techniques. The use of this method of data analysis allows direct comparison with the results of earlier, four slice coronary CTA studies. The results were then recalculated after exclusion of patients with $a \geqslant 400$ CAC score.

\section{RESULTS}

\section{Patients}

The multislice CTA procedure was universally well tolerated and no complications occurred. However, one patient moved significantly during the multislice CT acquisition and had to be excluded from the analysis. Therefore, 57 patients were evaluated fully. The time between conventional angiography and CTA was a mean (SD) of 9 (4) days. The average investigation time was less than 20 minutes although reconstruction took about another 5-7 minutes. Preparation and evaluation of the studies at the workstation took about 25 minutes. The mean (SD) heart rate recorded at the time of enrolment into the study was 61 (8) beats/min, whereas mean heart rate recorded during the actual multislice coronary CTA acquisition was 64 (15) beats/min (range 4090 beats/min). The probable reasons for this disparity are discussed below. 


\section{Cardiac catheterisation coronary angiography findings}

Of 741 possible coronary artery segments for analysis, the invasive coronary angiograms showed 22 segments containing stents and 40 segments downstream of total occlusions. There were four segments of a recessive right coronary artery. All of these 66 segments were excluded from the analysis. Therefore, 675 segments remained for the comparative evaluation. According to the invasive coronary angiograms 25 patients had normal coronary artery segments or no significantly diseased $(<50 \%)$ segments, nine patients had one vessel disease, 12 patients had two vessel disease, and 11 patients had three vessel disease. This corresponded to 90 significantly diseased coronary artery segments and 585 normal or not significantly diseased segments. The number of patients $(25 / 57,44 \%)$ without significant coronary artery disease was disproportionately high given that, for the purposes of this study, segments of coronary artery containing stents were not classified as containing a $>50 \%$ stenosis. The distribution of coronary artery segments containing $>50 \%$ lesions or occlusions was two in the left main stem, 31 in the left anterior descending artery, 30 in the circumflex, and 27 in the right coronary artery.

\section{Multislice coronary CTA findings}

Of the 90 severely diseased coronary artery segments, 75 were correctly diagnosed with coronary CTA (overall sensitivity $83 \%)$. Fifteen significantly stenosed coronary artery segments were not identified (false negative). Overall 19 segments were diagnosed as containing a significant stenosis although they did not (false positive). Therefore, of the 585 nonseverely diseased segments, 566 were correctly identified (overall specificity 97\%). The corresponding positive and negative predictive values were $80 \%$ and $97 \%$, respectively. Absence of significant coronary artery disease was correctly diagnosed in $96 \%$ (24 of 25 ) of patients. The false positive segments were almost universally restricted to the patients with severely diseased segments elsewhere. Of the 32 patients with significant coronary artery disease, none were categorised as normal and 27 (84\%) were appropriately categorised according to the number of diseased vessels. All occluded segments of coronary artery were correctly classified as being $>50 \%$ stenotic, but only half of these (seven of 14 ) were specifically identified as occlusions in view of collateral flow. The 22 segments of coronary artery containing stents were analysed separately from the main analysis. All the stents were accurately diagnosed as being patent by coronary CTA. Only one stent had significant $(>50 \%)$ in-stent restenosis and this was also accurately assessed with coronary CTA.

Overall 37\% (21 of 57) of patients had total CAC scores $>400$. Analysis was repeated with these patients excluded. Of the 38 remaining severely diseased coronary artery segments, 34 were correctly diagnosed with multislice CT

Table 2 Accuracy of submillimetre coronary CTA for diagnosing significantly diseased coronary artery segments for all patients (overall) and for those without significant coronary artery calcification (CAC score $<400$ )

\begin{tabular}{lll}
\hline & $\begin{array}{l}\text { Overall } \\
\text { group }\end{array}$ & CAC score $<\mathbf{4 0 0}$ \\
\hline Sensitivity & $83 \%$ & $89 \%$ \\
Specificity & $97 \%$ & $98 \%$ \\
Positive predictive value & $80 \%$ & $79 \%$ \\
Negative predictive value & $97 \%$ & $99 \%$ \\
\hline
\end{tabular}

(overall sensitivity $89 \%$ ). Nine segments were diagnosed as containing a significant stenosis although they did not. Therefore, of the remaining 417 non-severely diseased segments, 408 were correctly identified (specificity 98\%). The corresponding positive and negative predictive values were $79 \%$ and $99 \%$, respectively. Table 2 summarises the results of the overall and subgroup analyses.

\section{DISCUSSION}

In keeping with the findings presented by other authors, submillimetre multislice CT has been shown to offer major advantages over four slice CT for the evaluation of the coronary artery lumen. In this study it has been possible to perform coronary CTA with the realistic expectation that all major coronary artery segments and side branches would be assessable. The level of accuracy was generally high for the evaluation of these coronary artery segments, in comparison with invasive coronary angiography. These findings may be attributable predominantly to the technological advances outlined above and previous investigator experience of multislice coronary CTA.

However, previously published investigations have not made use of either a 16 slice acquisition or partial subsegmental reconstruction algorithms. It is therefore surprising that, at first glance, the overall sensitivity presented here for lesion detection appears to compare unfavourably with the previously published submillimetre multislice coronary CTA studies. ${ }^{78}$ In fact this is not the case. Closer analysis of the data shows that in both the previously published trials the coronary CTA data were analysed for each vessel rather than each segment. The justification for using this approach was cited as difficulty in assessing coronary artery segments downstream of coronary artery occlusions. Lack of validation for the American Heart Association segmental model of coronary artery anatomy when used for cardiac CT was cited as a further reason. ${ }^{11}$ Neither of these reasons are necessarily valid and make direct comparison with previous data difficult. This is a limitation of these studies. Indeed it is probable that the reported sensitivity for stenoses detection on a per vessel basis would be higher than that reported on a per segment analysis, and this likelihood needs to be considered when assessing the data presented here.

The relatively high number of patients (22) with heart rates $\geqslant 65$ beats/min during scan acquisition was surprising in view of the inclusion criteria. The reason for this became apparent at the end of the study. Patients with presumed coronary artery disease were taking oral $\beta$ blockers (or other heart rate slowing drugs) at the time of their invasive coronary angiogram. A number of patients had their drug treatment altered before coronary CTA. Often patients without significant coronary artery disease had even stopped taking their oral $\beta$ blockers. This not only resulted in a higher mean heart rate at $\mathrm{CT}$ than with invasive coronary angiography, but may have introduced a source of error in data analysis in the case of withdrawal of drug treatments inducing vasodilatation. This is a limitation of the study design. In retrospect stipulating no changes in treatment between the two investigations and giving additional $\beta$ blockers if necessary would have been preferable. It may be postulated that better heart rate control during CT acquisition for all patients would have affected the accuracy of the investigation beneficially.

There are other limitations to this investigation. If a low dose CAC score is to be used as a gatekeeper coronary CTA, then further work is needed to confirm exactly what tube current gives adequately reproducible CAC scores. Furthermore, different cut off CAC scores may need to be assessed for the subsequent accuracy of coronary CTA data. 
However, the cut off level should not be too low to rule out most patients but, by the same token, the investigation should be optimal in terms of diagnostic accuracy. The radiation exposure incurred in submillimetre multislice coronary CTA (effective dose estimated to be up to $10.0 \mathrm{mSv}$ ) remains a concern. Unfortunately ECG controlled tube current modulation (which gives greatly reduced dose during systole) is not available with all CT manufacturers at present and was not available for this study. The dose reduction strategies incorporated into some 16 slice scanners have prevented increases in the effective dose given by the four slice scanners and even decreased the dose. To put the patient radiation dose into context, if the investigation proposed supplies predictable, accurate diagnostic information about the coronary arteries then the effective radiation doses, with ECG controlled tube current modulation, are quite acceptable for clinical use $(4.3 \mathrm{mSv}) .{ }^{14}$ Indeed, under these circumstances, the dose compares favourably with that incurred during invasive coronary angiography.

Of note, in patients for whom a CAC score did not indicate severe calcification, the accuracy of evaluation of coronary artery segments was very high (sensitivity $89 \%$, specificity $98 \%$ ). The results presented here suggest that submillimetre multislice CT may allow avoidance of a first time invasive diagnostic coronary angiogram in selected patients. Further investigation with larger patient populations is needed. A protocol advocating adequate heart rate control and the recording of an initial CAC scoring scan to act as a gatekeeper for going on to coronary CTA may be proposed. Patients with high CAC scores would still require invasive angiography, whereas patients rendered adequately bradycardic with $\beta$ blockers who also have lower CAC scores would undergo coronary CTA. It seems probable that patients without significant coronary artery disease would be selected accurately, so avoiding invasive angiography. What remains to be determined is whether patients would not be disadvantaged, in terms of either hard end points or indeed symptoms, by such an approach. In view of the crucially important nature of the investigation such an approach should perhaps be recommended as the final step in validation of the technique before clinical use.

\section{Conclusion}

Submillimetre, retrospectively ECG gated coronary CTA with 16 slice CT reliably produces high quality images. Accurate quantitative analysis has been shown to be possible and it appears that a subgroup of patients may be selected, by using a CAC score, with even higher sensitivity and specificity. The findings of this investigation suggest that coronary CTA has a useful clinical role in the investigation of coronary heart disease. Submillimetre multislice coronary CTA should be further investigated with larger patient populations and excluding patients with significant CAC.

We thank the radiographers who kindly helped in the acquisition of all the CT data for this study.

\section{Authors' affiliations}

G J Morgan-Hughes, P E Owens, A J Marshall, South West Cardiothoracic Centre, Plymouth NHS Trust, Derriford, Plymouth, UK

C A Roobottom, Department of Radiology, Plymouth NHS Trust

Gareth Morgan-Hughes has received lecture fees from General Electrical Medical Systems, Milwaukee, USA.

\section{REFERENCES}

1 Achenbach S, Ulzheimer S, Baum U, et al. Noninvasive coronary angiography by retrospectively ECG-gated multislice spiral CT. Circulation 2000;102:2823-8.

2 Nieman K, Oudkerk M, Rensing B, et al. Coronary angiography with multislice computed tomography. Lancet 2001;357:599-603.

3 Knez A, Becker C, Leber A, et al. Usefulness of multislice spiral computed tomography angiography for determination of coronary artery stenoses. Am J Cardiol 2001;88:1191-4.

4 Morgan-Hughes G, Marshall A, Roobottom C. Multislice computed tomographic coronary angiography: experience in a UK centre. Clin Radiol 2002;58:378-83.

5 Kopp A, Schröder S, Küttner A, et al. Non-invasive coronary angiography with high resolution multidetector-row computed tomography: results in 102 patients. Eur Heart J 2002;23:1714-25.

6 Flohr T, Schoepf J, Küttner A, et al. Advances in cardiac imaging with 16section CT systems. Acta Radiol 2003;10:386-401.

7 Nieman K, Cademartiri F, Lemos P, et al. Reliable non-invasive coronary angiography with fast submillimetre multislice spiral computed tomography. Circulation 2002; 106:2051-4.

8 Ropers D, Baum U, Pohle K, et al. Detection of coronary artery stenosis with thin-slice multi-detector row spiral computed tomography and multiplanar reconstruction. Circulation 2003;107:664-6.

9 Heuschmid M, Kuttner A, Flohr T, et al. [Visualisation of coronary arteries in $\mathrm{CT}$ as assessed by a new 16 slice technology and reduced gantry rotation time: first experiences]. Rofo Fortschr Geb Rontgenstr Neven Bildgeb Verfahr 2002;174:721-4

10 Rumberger J. Noninvasive coronary angiography using computed tomography. Ready to kick it up a notch? Circulation 2002;106:2036-8.

11 Austen W, Edwards J, Frye R, et al. A reporting system on patients evaluated for coronary artery disease. Circulation 1975;51:5-40.

12 Rumberger J, Brundage B, Rader D, et al. Electron beam computed tomographic coronary calcium scanning: a review and guidelines for use in asymptomatic persons. Mayo Clin Proc 1999:74:243-52.

13 Agatston A, Janowitz W, Hildner F, et al. Quantification of coronary artery calcium using ultrafast computed tomography. J Am Coll Cardiol 1990;15:827-32.

14 Jakobs T, Becker C, Ohnesorge B, et al. Multislice helical CT of the heart with retrospective ECG gating: reduction of radiation exposure by ECG-controlled tube current modulation. Eur Radiol 2002; 12:1081-6.

\section{ELECTRONIC PAGES}

\section{Heart Online case reports: www.heartinl.com}

The following electronic only article is published in conjunction with this issue of Heart.

\section{An unusual cause of abrupt vessel closure J B Foley, J Cosgrave, D S O'Briain}

A 60 year old woman presented with chest pain. An ECG showed ST depression across the anterior leads and lateral $\mathrm{T}$ wave inversion and angiography showed a significant proximal circumflex lesion. After percutaneous intervention to the circumflex artery she had a cardiac arrest and died.
Postmortem examination found a stent blocked with a combination of thrombus and a tangle of translucent material. Embolic coronary artery occlusion is well described but this is the first report of embolisation of material arising from the lining of the guiding catheter as the cause.

(Heart 2005;91:e19) www.heartjnl.com/cgi/content/full/91/ $3 / \mathrm{e} 19$ 